KIAS-P98019

hep-th/9808183

August 1998

\title{
Effective Action for Membrane Dynamics in DLCQ $M$ theory on a Two-torus
}

\author{
Seungjoon Hyun[, Youngjai Kiem[], and Hyeonjoon Shin[ \\ School of Physics, Korea Institute for Advanced Study, Seoul 130-012, Korea
}

\begin{abstract}
The effective action for the membrane dynamics on the background geometry of the $N$-sector DLCQ $M$ theory compactified on a two-torus is computed via supergravity. We compare it to the effective action obtained from the matrix theory, i.e., the $(2+1)$ dimensional supersymmetric Yang-Mills (SYM) theory, including the one-loop perturbative and full non-perturbative instanton effects. Consistent with the DLCQ prescription of $M$ theory a la Susskind, we find the precise agreement for the finite $N$-sector (offconformal regime), as well as for the large $N$ limit (conformal regime), providing us with a concrete example of the correspondence between the matrix theory and the DLCQ $M$ theory. Non-perturbative instanton effects in the SYM theory conspire to yield the eleven-dimensionally covariant effective action.
\end{abstract}

\footnotetext{
${ }^{1}$ hyun@kiasph.kaist.ac.kr

${ }^{2}$ ykiem@kiasph.kaist.ac.kr

${ }^{3}$ hshin@kiasph.kaist.ac.kr
} 
The original formulation of the matrix theory, a promising candidate for the quantum description of $M$ theory, is given in the infinite momentum frame (IMF) requiring us to consider the large parton number limit [1]. Susskind then proposed the discrete light-cone quantization (DLCQ) version of the matrix theory where one considers the light-like eleventh direction, which was suggested to define the matrix theory in the finite $N$-sector [2]. Becker, Becker, Polchinski, and Tseytlin considered the scattering between two $D$-particles ( $M$-momentum) and showed that the matrix side calculation for the effective action precisely reproduces the eleven-dimensional supergravity side calculation up to two loops [3]. To find the precise agreement, it is indeed necessary that the background geometry produced by the source $M$-momentum should not be taken as the usual dimensionally uplifted version of the $D$-particle solution along the spatial $M$ theory circle but as the DLCQ version uplifted along the light-cone circle [4]. As advocated in [5] and further elaborated in [6], this tells us that the DLCQ $M$ theory should be compared with the $M$ theory on a non-trivial background rather than on a flat background. Along this line of idea, the same type of background geometry as in [4] was utilized in [7] to show the agreement between the DLCQ supergravity and the matrix theory for three-graviton scatterings (see also [8]).

For the DLCQ $M$ theory compactified on a two-torus, the microscopic description via the matrix theory is given by the $(2+1)$-dimensional supersymmetric Yang-Mills theory [9, 10]. Taking the same DLCQ prescription for the background geometry of the $N$-sector DLCQ $M$ theory on a two-torus yields a non-asymptotically flat background space-time with the asymptotic geometry of the Anti de Sitter (AdS) type [5, 6]. In the large $N$ and the large eleventh radius $R$ (decompactification) limit while $N / R$ being fixed, the background geometry becomes a tensor product of an AdS space and a sphere, i.e., $A d S_{4} \times S^{7}$. In the same limit, the matrix theory description turns to a conformal field theory (CFT) as the infrared limit of SYM [6]. This is the case where the holographic AdS/CFT correspondence is conjectured to be valid [11, [12]. According to the motivation of the DLCQ $M$ theory [2], one might hope further that the correspondence between the matrix theory and the DLCQ $M$ theory on the background geometry of [0, [6] persists even in the case of the finite $N$ and the finite $R$. In this paper, we find an explicit example that 
directly supports this idea; we compute the effective action for the membrane dynamics on the background geometry of the DLCQ $M$ theory on a two-torus via supergravity for the finite value $N$ and $R$. We then show that the effective action up to the fourth order in the membrane velocity exactly reproduces the effective action computed from the $(2+1)$ dimensional SYM including the one-loop perturbative and full non-perturbative instanton effects for the finite value of $N$ and $R$, which were recently calculated in [13. Our effective action also behaves smoothly as we take the limit where $N$ and $R$ goes to infinity while the ratio being fixed. Our analysis shows that the instantons in the matrix theory, the $(2+1)$-dimensional SYM that does not have a manifest eleven-dimensional covariance, conspire to yield the results that derive from the manifestly covariant eleven-dimensional DLCQ supergravity on the non-asymptotically flat background geometry.

In the case of the small value of $R$, the membrane dynamics in string/supergravity theory and its comparison to the matrix theory have been discussed in the literature [14] [20]. In [16], D2-brane scatterings in the asymptotically flat ten-dimensional background geometry were found to be in agreement with the perturbative one-loop SYM. Polchinski and Pouliot [17] added the one-instanton correction to the effective action to investigate the effect of the $M$-momentum transfer and found an approximate agreement with the eleven-dimensional supergravity result. The SYM side analysis was generalized in 18 to include the semi-classical multi-instanton effect. A clear physical interpretation of the instanton effects is also given in [19]. In addition to the small $R$ limit, we note that by adopting the asymptotically flat background geometry, it was necessary to take the large $N$ limit to find the agreement in [16 - [19], just as what happens in the IMF formulation of [1]. In the context of the DLCQ $M$ theory, an agreement for the finite $N$ and the small value of $R$ for the membrane dynamics was observed in [20]. In the limit of small $R$, the instanton corrections are suppressed and the agreement in 20] is up to the perturbative one-loop effect in SYM. The salient feature of our result presented here is the removal of the restriction to the large $N$ and small $R$. By adopting the background geometry of the finite $N$-sector DLCQ $M$ theory proposed in [5, 6] the former restriction is removed. Furthermore, by considering the full non-perturbative instanton corrections on the SYM side, we are able to find the agreement for an arbitrary value of $R$. The conventional 
range of the validity for the AdS/CFT correspondence is the large $R$ and the large $N$ limit while the ratio being fixed [1].

The background geometry of $N$-sector DLCQ $M$ theory compactified on a two-torus is given by the following eleven-dimensional metric [5, 6]

$$
d s_{11}^{2}=h^{-2 / 3}\left(-d t^{2}+d x_{8}^{2}+d x_{9}^{2}\right)+h^{1 / 3}\left(d x_{1}^{2}+\cdots+d x_{7}^{2}+d x_{11}^{2}\right),
$$

where the eleven-dimensional harmonic function $h$ is given by

$$
h=\sum_{n=-\infty}^{\infty} \frac{\kappa N}{\left(r^{2}+\left(x_{11}+2 \pi R n\right)^{2}\right)^{3}} .
$$

We introduce $r^{2}=x_{1}^{2}+\cdots+x_{7}^{2}$ and the torus extends over the $x_{8}$ and $x_{9}$ directions. The integer $N$ represents the number of the coincident source membranes and $\kappa$ is a dimensionful constant. The eleventh circle along the $x_{11}$ coordinate has the radius $R$ and the function $h$ contains the contribution from all possible mirror charges to respect the periodicity under the lattice translation $x_{11} \rightarrow x_{11}+2 \pi R$. In the limit of large $R$, which corresponds to the decompactification limit of the DLCQ $M$ theory, the metric (而) becomes that of the $A d S_{4} \times S^{7}$; the eleventh direction becomes indistinguishable from other noncompact directions $\left(x_{1}, \cdots, x_{7}\right)$ and the transversal $S O(7)$ symmetry gets enhanced to the $S O(8)$ symmetry [21]. Specifically, the summation in the expression for $h$ gets dominated by the $n=0$ term, which has the manifest $S O(8)$ symmetry. This is the limit where we have the large $N$ AdS/CFT correspondence, where the AdS supergravity and the CFT near the infrared fixed point, i.e., the conformal phase of the (2+1)-dimensional SYM, become a dual description to each other. In the limit of the vanishingly small $R$, we can replace the summation in Eq. (2) with an integration and recover the near-horizon geometry of the $N D 2$-branes of the type IIA supergravity. Our primary interest will be the case of the arbitrary values of $R$ and $N$. For the purpose of the comparison to the $(2+1)$-dimensional SYM, which is the dimensional reduction of the ten-dimensional SYM, it is convenient to reshuffle the series summation of Eq. (21) using the Poisson resummation formula:

$$
\sum_{n=-\infty}^{\infty} f(n)=\sum_{m=-\infty}^{\infty} \int_{-\infty}^{\infty} d \phi f(\phi) e^{2 \pi i m \phi}
$$


The resummation can be exactly performed to result the following identity.

$$
\begin{gathered}
\sum_{n=-\infty}^{\infty} \frac{1}{\left(r^{2}+\left(x_{11}+2 \pi R n\right)^{2}\right)^{3}}=\frac{1}{16 R}\left[\frac{3}{r^{5}}+\frac{1}{r^{3}} \sum_{m=1}^{\infty} \frac{m^{2}}{R^{2}} e^{-m r / R} 2 \cos \left(m x_{11} / R\right)\right. \\
\left.+\frac{3}{r^{4}} \sum_{m=1}^{\infty} \frac{m}{R} e^{-m r / R} 2 \cos \left(m x_{11} / R\right)+\frac{3}{r^{5}} \sum_{m=1}^{\infty} e^{-m r / R} 2 \cos \left(m x_{11} / R\right)\right] .
\end{gathered}
$$

We recognize the first term on the right hand side (RHS) as having the structure of the perturbative one-loop term in the $D 2$-brane effective action [16. The summation index $m$ on the RHS appears in the form of the $M$-momentum $m / R$. According to the argument of Ref. [17], the integer $m$ may be interpreted as the measure of the $M$-momentum transfer between the source and probe membranes. The key observation for our later purpose is that the three infinite series terms on the RHS can be rewritten in terms of the modified Bessel function $K_{\nu}$ with a half-integer $\nu$, which has the finite number of terms in an expansion 22

$$
K_{j+1 / 2}(z)=\left(\frac{\pi}{2 z}\right)^{\frac{1}{2}} e^{-z} \sum_{k=0}^{j} \frac{(j+k) !}{k !(j-k) !(2 z)^{k}} .
$$

The function $h$ can then be succinctly written as

$$
h\left(r, x_{11}\right)=\frac{\kappa N}{16 R}\left[\frac{3}{r^{5}}+\sum_{m=1}^{\infty}\left(\frac{2}{\pi}\right)^{\frac{1}{2}} \frac{m^{2} m^{1 / 2}}{R^{5}}\left(\frac{R}{r}\right)^{5 / 2} K_{5 / 2}(m r / R) 2 \cos \left(m x_{11} / R\right)\right] .
$$

We now consider the dynamics of a probe membrane, which is taken to be spanning the $x_{8}, x_{9}$ directions and is moving with a constant velocity $v_{i}=\partial_{0} x_{i}$ in a direction transversal to the probe and $x_{11}$, i.e., $i=1, \cdots, 7$. The action for the probe membrane is

$$
S_{2}=-T_{2} \int d^{3} x \sqrt{-\operatorname{det} h_{\mu \nu}}+i \mu_{2} \int H
$$

where $T_{2}$ is the membrane tension and $H$ is the three-form gauge field of the elevendimensional supergravity. The metric $h_{\mu \nu}$ is the induced metric on the world-volume of the probe membrane given by

$$
h_{\mu \nu}=g_{\mu \nu}+\partial_{\mu} x^{I} \partial_{\nu} x^{J} g_{I J}
$$

Here $\mu, \nu$ are the world-volume indices and $I, J$ are the indices for the transverse directions to the probe. We plug the metric Eq. (1) with the function $h$ of Eq. (6) into the 
action $S_{2}$ and expand it in powers of the transverse velocity $v$. The action $S_{2}$ becomes

$$
S_{2}=\int d^{3} x\left[\frac{1}{2} T_{2} v^{2}-V_{2}+\mathcal{O}\left(\left(v^{2}\right)^{3}\right)\right]
$$

where $V_{2}$ is the interaction potential given by

$$
\begin{aligned}
V_{2}= & -\frac{1}{8} T_{2} h\left(r, x_{11}\right)\left(v^{2}\right)^{2} \\
= & -\frac{N}{16 R M_{p}^{3}}\left(v^{2}\right)^{2} \\
& \times\left[\frac{3}{r^{5}}+\sum_{m=1}^{\infty}\left(\frac{2}{\pi}\right)^{\frac{1}{2}} \frac{m^{2} m^{1 / 2}}{R^{5}}\left(\frac{R}{r}\right)^{5 / 2} K_{5 / 2}(m r / R) 2 \cos \left(m x_{11} / R\right)\right] .
\end{aligned}
$$

Going to the last line, we use the fact that $T_{2} \kappa=8 M_{p}^{-3}$ where $M_{p}$ is the eleven-dimensional Planck scale [17. It should be noted that the potential is valid for any value of $R$. If the value of $R$ is very small (or $r \gg R$ ) and we take $m=1$, the potential is approximated by

$$
V_{2} \approx-\frac{3 N}{16 R M_{p}^{3}} \frac{\left(v^{2}\right)^{2}}{r^{5}}-\frac{N}{16 R^{3} M_{p}^{3}} \frac{\left(v^{2}\right)^{2}}{r^{3}} e^{-r / R} 2 \cos \left(x_{11} / R\right) .
$$

The first term on the RHS is the usual potential between two D2-branes in the tendimensional type IIA theory [16] and the second term is the potential due to the effect of a single $M$-momentum transfer [17]. The approximate potential Eq. (11) shows a notable feature that there is no $r$ independent $v^{4}$ term that appeared in [17. Under the large $N$ limit, it is natural to drop this term as was done in, for example, [16]. In the DLCQ framework, this term is automatically absent [4, 20], and this feature is also present in the case of the exact potential, Eq. (10).

According to the prescription of Seiberg and Sen, the DLCQ $M$-theory on a two-torus is described by a system of $D 2$-branes wrapped on its $T$-dual two-torus [10. When the number of $D 2$-branes is $N$, the action for the system is just the $(2+1)$-dimensional $U(N)$ SYM theory. The interaction potential between the source and the probe membranes is given by the effective potential of the SYM theory, and we compare the supergravity effective potential Eq. (10) to the effective potential of the SYM theory. We note that our supergravity side calculation is actually for the two-body dynamics of the source and the probe. This enables us to restrict our attention only to the SU(2) SYM theory, as far as the dynamics is concerned. From the gauge theory point of view, we do not give 
the vacuum expectation values to the scalars that represent the position of the $N$ source membranes, thereby making them localized at one transversal space-time point. In what follows, we thus put the factor $N$ in front of the effective action and omit the trace part [16, 20]. We note that to tackle the genuine $N$-body dynamics, we have to use the full $S U(N)$ SYM theory and this problem is the beyond of scope of this paper.

Generically, the one-loop effective action $\Gamma^{(1)}$ of the SYM theory is the summation of a perturbative term and $m$-instanton terms. Hereafter, for the notational convenience, we call the perturbative term 0-instanton sector. The general structure of the effective action [23, 24] looks schematically like

$$
\Gamma^{(1)}=N \int d^{3} x\left(f^{(0)} u^{4}+f^{(2)} u^{3}\left[\psi^{2}\right]+f^{(4)} u^{2}\left[\psi^{4}\right]+f^{(6)} u\left[\psi^{6}\right]+f^{(8)}\left[\psi^{8}\right]\right)
$$

where $u^{i}=\dot{\phi}^{i}=F_{0 i}$, the $i$-th component of the electric field, $\left[\psi^{p}\right]$ denotes a generic $p$ fermion structure, and $f^{(p)}$ represents the bosonic coefficient function of the corresponding $p$ fermion structure. The scalars $\phi^{i}(i=1, \cdots, 7)$ are the seven scalars of the vector multiplet (thereby having the $S O(7)$ symmetry). The function $f^{(p)}$ consists of the instanton summation and we represent it as $f^{(p)}=\sum_{m=-\infty}^{\infty} f_{m}^{(p)}$ where $m$ is the instanton number. The bosonic zero fermion term $f^{(0)}$ should be compared to what we found on the supergravity side analysis. Recently, Paban, Sethi and Stern [13 exactly computed the eight fermion terms; $\left[\psi^{8}\right]$ consists of terms with zero, two and four scalar structure, and they are determined via the supersymmetry by requiring the absence of nine fermion terms under the supersymmetry transformation. Once $f^{(8)}$ term is calculated from their analysis, the remaining terms are determined by the supersymmetric completion. To ensure the supersymmetry, we have

$$
\delta \phi^{i}\left(\frac{\partial}{\partial \phi^{i}} f^{(p)}\right)\left[\psi^{p}\right]_{\max }=\delta \phi^{i} \phi^{i}\left(\frac{d}{\phi d \phi} f^{(p)}\right)\left[\psi^{p}\right]_{\max }=-f^{(p+2)} \delta_{\psi}\left[\psi^{p+2}\right]_{\max },
$$

where $\delta_{\psi}$ is only for the variation of fermion fields. Here, $\left[\psi^{p}\right]_{\max }$ denotes the $p$ fermion term with the maximum number scalar structure. We note that the function $f^{(p)}$ depends only on the $S O(7)$ invariant combination $\phi^{2}=\phi^{i} \phi^{i}$. Thus, the function $f_{m}^{(0)}$ is related to $f_{4, m}^{(8)}$ by

$$
\left(\frac{d}{\phi d \phi}\right)^{4} f_{m}^{(0)}=k f_{4, m}^{(8)}
$$


where $f_{4, m}^{(8)}$ is the coefficient function of the four scalar structure term among $\left[\psi^{8}\right]$ in the $m$-instanton sector

$$
\sum_{m=-\infty}^{\infty} f_{4, m}^{(8)}\left(\phi^{i} \phi^{j} \phi^{k} \phi^{l} T^{i j k l}\right)
$$

Here $k$ is a constant and $T^{i j k l}$ is the eight fermion structure. From [13, $f_{4, m}^{(8)}$ is given by

$$
f_{4, m}^{(8)}=m^{6}|m|^{1 / 2} \frac{1}{g_{\mathrm{YM}}^{28}}\left(\frac{g_{\mathrm{YM}}^{2}}{\phi}\right)^{13 / 2} K_{13 / 2}\left(|m| \phi / g_{\mathrm{YM}}^{2}\right) e^{i m \phi^{8} / g_{\mathrm{YM}}^{2}}
$$

up to the overall multiplicative constant. The extra scalar $\phi^{8}$ is the dual magnetic scalar and $g_{\mathrm{YM}}$ is the three-dimensional Yang-Mills coupling constant. We remark that Eq. (16) gives the perturbative term when we set $m=0$ proportional to $\phi^{-13}$. Noting 22

$$
\left(\frac{d}{z d z}\right)^{a}\left(z^{-\nu} K_{\nu}(z)\right)=(-1)^{a} z^{-\nu-a} K_{\nu+a}(z)
$$

we conclude

$$
f_{m}^{(0)}=C m^{2}|m|^{1 / 2} \frac{1}{g_{\mathrm{YM}}^{12}}\left(\frac{g_{\mathrm{YM}}^{2}}{\phi}\right)^{5 / 2} K_{5 / 2}\left(|m| \phi / g_{\mathrm{YM}}^{2}\right) e^{i m \phi^{8} / g_{\mathrm{YM}}^{2}}
$$

from Eq. (14), where $C$ is a overall constant. The constant $C$ can not be determined by the argument of [13, but the one-instanton calculation of [17] determines it to be $C=(2 / \pi)^{1 / 2} g_{\mathrm{YM}}^{2} / 16$. Thus, the bosonic one-loop effective action $\Gamma_{B}^{(1)}$ from the SYM theory is

$$
\Gamma_{B}^{(1)}=N \int d^{3} x \sum_{m=-\infty}^{\infty} f_{m}^{(0)}\left(u^{2}\right)^{2}
$$

including the full non-perturbative instanton corrections. Since $\Gamma_{B}^{(1)}=-\int d^{3} x V_{\mathrm{SYM}}$, the effective potential $V_{\mathrm{SYM}}$ is

$$
\begin{aligned}
V_{\mathrm{SYM}} & =-\frac{N}{16}\left(u^{2}\right)^{2} \\
& \times\left[\frac{3}{\phi^{5}}+\sum_{m=1}^{\infty}\left(\frac{2}{\pi}\right)^{\frac{1}{2}} \frac{m^{2} m^{1 / 2}}{g_{\mathrm{YM}}^{10}}\left(\frac{g_{\mathrm{YM}}^{2}}{\phi}\right)^{5 / 2} K_{5 / 2}\left(m \phi / g_{\mathrm{YM}}^{2}\right) 2 \cos \left(m \phi^{8} / g_{\mathrm{YM}}^{2}\right)\right]
\end{aligned}
$$

We note that Eq. (20) is exactly identical to Eq. (10) if we identify $\phi_{i}=x_{i} / l_{s}^{2}, \phi^{8}=x_{11} / l_{s}^{2}$, $u=v / l_{s}^{2}$, and use $g_{\mathrm{YM}}^{2}=g_{s} / l_{s}$. The string coupling constant $g_{s}$ and the string length scale $l_{s}$ are related to the $M$ theory quantities by $g_{s}=\left(R M_{p}\right)^{3 / 2}$ and $l_{s}=\left(R M_{p}^{3}\right)^{-1 / 2}$. 
We thus constructed an explicit example that shows the agreement between the matrix theory and the DLCQ $M$ theory on the non-asymptotically flat background geometry of [0], which is consistent with the finite $N$-sector DLCQ prescription of [2]. This example points to the possibility that, at the level of finite $N$, the AdS/CFT correspondence may be elevated to the dual description via the matrix theory of the $M$ theory on the nonasymptotically-flat background geometry of [5]; a qualitative argument toward this effect has already been given in [6].

Regarding the matrix theory itself, our result clarifies the space-time aspects; for example, the locality along the $M$ theory circle and the eleven-dimensional covariance are not at all manifest from the point of view of the matrix theory. Turning our calculations around, the effective action of the (2+1)-dimensional SYM theory Eq. (19) can be Poissonresummed back to yield a eleven-dimensional harmonic function proportional to Eq. (2). At the level of the finite $R$, only the $S O(7)$ symmetry is manifest. As we take the limit where $R \rightarrow \infty$ and $N \rightarrow \infty$ while keeping the ratio $N / R$ fixed (the IMF limit), however, Eq. (2) shows that the symmetry enhances to the $S O(8)$ symmetry ( $n=0$ term) and the $(2+1)$-dimensional magnetic scalar combines with seven other scalars to yield the eight coordinates transversal to the $M$ membranes. This is the expected covariance for the $M$ theory compactified on a two-torus. To recover the eleven-dimensional covariance, the full non-perturbative effects on the gauge theory side should be taken into account.

\section{References}

[1] T. Banks, W. Fischler, S. H. Shenker, L. Susskind, Phys. Rev. D 55, 5112 (1997).

[2] L. Susskind, hep-th/9704080.

[3] K. Becker, M. Becker, J. Polchinski, A. Tseytlin, Phys. Rev. D 56, 3174 (1997), hep-th/9706072.

[4] S. Hyun, Y. Kiem and H. Shin, Phys. Rev. D 57, 4856 (1998), hep-th/9712021.

[5] S. Hyun, to appear in Phys. Lett. B, hep-th/9802026

[6] S. Hyun and Y. Kiem, hep-th/9805136. 
[7] J. McCarthy, L. Susskind and A. Wilkins, hep-th/9806136.

[8] W. Taylor and M. Van Raamsdonk, hep-th/9806066; Y. Okawa and T. Yoneya, hepth/9806108.

[9] W. Taylor, Phys. Lett. B 394, 283 (1997), hep-th/9611042.

[10] N. Seiberg, Phys. Rev. Lett. 79, 3577 (1997), hep-th/9710009; A. Sen, Adv. Theor. Math. Phys. 2, 51 (1998), hep-th/9709220.

[11] J. Maldacena, hep-th/9711200.

[12] S. Gubser, I. Klebanov and A. Polyakov, Phys. Lett. B 428, 105 (1998), hepth/9802109; E. Witten, hep-th/9802150.

[13] S. Paban, S. Sethi, and M. Stern, hep-th/9808119.

[14] J. Maldacena, Nucl. Phys. Proc. Suppl. 68, 17 (1998), hep-th/9709099.

[15] A. A. Tseytlin, Nucl. Phys. Proc. Suppl. 68, 99 (1998), hep-th/9709123.

[16] G. Lifschytz and S. D. Mathur, Nucl. Phys. B499, 283 (1997), hep-th/9612087; O. Aharony and M. Berkooz, Nucl. Phys. B491, 184 (1997), hep-th/9611215.

[17] J. Polchinski and P. Pouliot, Phys. Rev. D 56, 6601 (1997), hep-th/9704029.

[18] N. Dorey, V. V. Khoze, and M. P. Mattis, Nucl. Phys. B 502, 94 (1997), hepth/9704197.

[19] E. Keski-Vakkuri and P. Kraus, hep-th/9804067.

[20] I. Chepelev and A. A. Tseytlin, Nucl. Phys. B 524, 69 (1998), hep-th/9801120.

[21] N. Itzhaki, J. Maldacena, J. Sonnenschein, and S. Yankielowicz, Phys.Rev. D 58, 046004 (1998), hep-th/9802042.

[22] I. S. Gradshteyn and I. M. Ryzhik, Table of Integrals, Series, and Products, 5th ed. (Academic Press, 1994). 
[23] J. A. Harvey, Nucl. Phys. Proc. Suppl. 68, 113 (1998), hep-th/9706039.

[24] J. F. Morales, C. A. Scrucca, and M. Serone, hep-th/9801183. 